\title{
$\alpha-\mathrm{Cu}_{2} \mathrm{Se}$ 有序起伏结构的原子级分辨率表征
}

彭海琳

北京大学化学与分子工程学院, 北京 100871

\section{Atomic Resolution Characterization of Ordered Fluctuation Structure in $\alpha-\mathrm{Cu}_{2} \mathrm{Se}$}

\section{PENG Hailin}

College of Chemistry and Molecular Engineering, Peking University, Beijing 100871, P. R. China.

Email: hlpeng@pku.edu.cn

微观结构与性能的关联是如今材料物理化学 研究的核心问题之一。自从德国物理学家 Knoll 和 Ruska $^{1}$ 在 1932 年成功制成了世界上第一台实用 化的透射电子显微镜(TEM), 电镜就成为了分析材 料微观结构不可或缺的有力工具。由于电磁透镜 仅具有凸透镜的特性, 无法通过和凹透镜的组合 来消除像差, 尤其是由于电磁透镜中近轴和远轴 磁场对电子束会聚能力不同导致的球差难以消 除。早在 1947 年, Scherzer ${ }^{2}$ 就提出了用多极电磁 系统校正球差的方案, 但受限于多极校正系统的 制造难度和当时的计算机处理能力, 对于球差的 校正一直是可望而不可及。随着技术的不断发展, Haider 等 3 终于在 1998 年成功制造出了针对 200

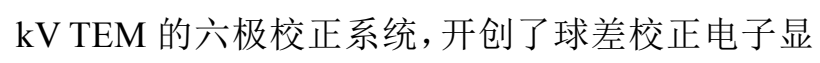
微术实用化的阶段。

$\mathrm{Cu}_{2} \mathrm{Se}$ 作为一种半导体热电材料很早就受到 了人们的关注。由于其热电性能在相变阶段有着 明显的提升, 热电优值 $(Z T)$ 出现了超过了 2 的峰 值 ${ }^{1}$, 这一材料的实用价值迅速凸显。然而对于其 低温 $\alpha$ 相的结构至今没有完善的解析, 这也成为 了进一步认识其性能提升机理的阻碍。早期受限 于表征手段的精度, 尽管许多研究 4-6 都注意到了 $\alpha-\mathrm{Cu}_{2} \mathrm{Se}$ 中的有序结构, 但更多的细节仍不明朗。 随着电镜技术的发展, Liu 等 7 于 2013 年首先获 得了沿 $<\overline{1} 10>_{\mathrm{c}}$ 带轴的球差校正扫描透射电子显微 镜(STEM)照片, 直接证明了其中的 $\mathrm{Se}-\mathrm{Cu}-\mathrm{Se}$ 层状 结构。随后 $\mathrm{Lu}$ 等 ${ }^{8}$ 在 2015 年利用电子衍射和 TEM 对其中的结构多形性, 尤其是沿 $<112>_{\mathrm{c}}$ 带轴的结
构起伏和演变做出了进一步探究。但对于衍射中 反映出的种种结构细节尚没有直接的原子级分辨 观察证据, 且众多结构模型也难以完全反映这一 结构的复杂性。鉴于该材料的重要性, 浙江大学材 料学院陈陆、王勇、张泽等人利用球差校正 STEM 对 $\alpha-\mathrm{Cu}_{2} \mathrm{Se}$ 的精细结构进行了原子级别的表征和 分析。

该工作已在物理化学学报上在线发表 (doi: 10.3866/PKU.WHXB201805111) ${ }^{9}$ 。文章首先 利用 FIB 技术制备了特殊取向的 $\alpha-\mathrm{Cu}_{2} \mathrm{Se}$ 样品, 并利用电子衍射对其结构进行了系统表征。然后 作者利用 STEM 首次获得了 $\alpha-\mathrm{Cu}_{2} \mathrm{Se}[\overline{1} 12]_{\mathrm{c}}$ 带轴的 原子级分辨高角环形暗场(HAADF)像, 并对该带 轴下新发现的复杂微观结构单元进行了详细分 析, 建立了对应其不同组成单元的若干种结构模 型。进一步, 作者基于建立的结构模型进行了 HAADF 像模拟, 获得了与实验像一致的结果。该 工作对于更为深入地理解 $\alpha-\mathrm{Cu}_{2} \mathrm{Se}$ 的复杂结构, 并进一步理解相变时体现出的优异热电性能提供 了重要信息。

\section{References}

(1) Knoll, M.; Ruska, E. Z. Phys. 1932, 78 (5-6), 318. doi: $10.1007 /$ bf01342199

(2) Scherzer, O. Optik 1947, 2, 114.

(3) Haider, M.; Rose, H.; Uhlemann, S.; Schwan, E.; Kabius, B.; Urban, K. Ultramicroscopy 1998, 75 (1), 53. doi: 10.1016/s0304-3991(98)00048-5 
(4) Milat, O.; Vucic, Z.; Ruscic, B. Solid State Ion. 1987, 23 (1-2), 37. doi: 10.1016/0167-2738(87)90079-8

(5) Kashida, S.; Akai, J. J. Phys. C 1988, 21 (31), 5329. doi: $10.1088 / 0022-3719 / 21 / 31 / 004$

(6) Frangis, N.; Manolikas, C.; Amelinckx, S. Phys. Status Solidi A 1991, 126 (1), 9. doi: 10.1002/pssa.2211260102

(7) Liu, H. L.; Yuan, X.; Lu, P.; Shi, X.; Xu, F. F.; He, Y.; Tang, Y. S.; Bai, S. Q.; Zhang, W. Q.; Chen, L. D.; et al. Adv. Mater. 2013, 25
(45), 6607. doi: 10.1002/adma.201302660

(8) Lu, P.; Liu, H. L.; Yuan, X.; Xu, F. F.; Shi, X.; Zhao, K. P.; Qiu, W. J.; Zhang, W. Q.; Chen, L. D. J. Mater. Chem. A 2015, 3 (13), 6901. doi: $10.1039 / \mathrm{c} 4 \mathrm{ta} 07100 \mathrm{j}$

(9) Chen, L; Liu, J; Wang, Y; Zhang, Z. Acta Phys. -Chim. Sin. 2019, 35 (2), 141. [陈陆, 刘军, 王勇, 张泽. 物理化学学报, 2019, 35 (2), 141.] doi: 10.3866/PKU.WHXB201805111 